\title{
NOTAS
}

\section{Hongos endófitos antárticos como herramienta para la reintroducción de especies nativas en zonas áridas}

\author{
Antarctic fungal endophytes as tool for the reintroduction of native plant species in arid zones
}

\author{
Cristian Fardella a ${ }^{\text {, Rómulo Oses }}{ }^{\text {a }}$, Cristian Torres-Díaz ${ }^{\text {b }}$, Marco A Molina-Montenegro a*

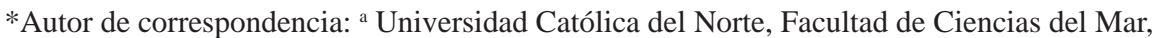 \\ Centro de Estudios Avanzados en Zonas Áridas (CEAZA), Coquimbo, Chile, marco.molina@ceaza.cl \\ b Universidad del Bío-Bío, Facultad de Ciencias, Departamento de Ciencias Básicas, Chillán, Chile.
}

\begin{abstract}
SUMMARY
Ecological restoration is the deliberate action that speeds recovery from a degraded system by direct or indirect human intervention. Xerophytic formations are among the most degraded ecosystems where low water availability makes reintroduction plans unsuccessful and extremely expensive. Roots inoculation with endophytic fungi has been reported as a successful strategy to maintain or improve the ecophysiological performance and survival in different species of shrubs and trees. In harsh environments, endophytes have been shown to provide benefits in terms of survival and growth for their associated vegetation. In this study, we showed that inoculation with endophytes isolated from plants growing in Antarctica improve survival and water use efficiency for irrigation on native species of xerophytic formations: Flourensia thurifera, Senna cumingii and Puya berteroniana. Overall, the presence of endophytes improved survival of all native species. Furthermore, the presence of endophytes improved water use efficiency for irrigation, reaching higher percentages of survival in plants with a reduction in the added water. The application of Antarctic endophytes could be considered a successful strategy for future projects and reintroduction programs in arid and semi-arid zones of Chile.
\end{abstract}

Key words: endophytes, water use efficiency, xerophytic formations, restoration.

\section{RESUMEN}

La restauración ecológica es la actividad deliberada que acelera la recuperación de un sistema degradado por acción directa o indirecta de la intervención humana. Las zonas xerofíticas están entre los ecosistemas más degradados donde la baja disponibilidad hídrica hace que los planes de reintroducción sean poco exitosos y extremadamente costosos. La aplicación de hongos endófitos a raíces ha sido reportada como una exitosa estrategia para mantener o mejorar el desempeño ecofisiológico y supervivencia en diferentes especies de arbustos y árboles. En ambientes climáticamente extremos, los endófitos han mostrado proporcionar beneficios en términos de supervivencia y crecimiento a la vegetación que se asocia a ellos. En el presente trabajo mostramos como la inoculación con endófitos aislados desde plantas creciendo en la Antártica mejoran la supervivencia y eficiencia en el uso del agua para el riego en especies nativas de las formaciones xerofíticas: Flourensia thurifera, Senna cumingii y Puya berteroniana. En general, los resultados muestran que la presencia de endófitos mejoró la supervivencia de las tres especies nativas. Por otro lado, la presencia de endófitos mejoró la eficiencia en el uso del agua para el riego, permitiendo alcanzar altos porcentajes de supervivencia en plantas con una reducción en el agua requerida. Los resultados preliminares obtenidos en este estudio sugieren que la aplicación de endófitos antárticos podría ser considerada como una estrategia exitosa para proyectos futuros y programas de reintroducción en zonas áridas y semi-áridas de Chile.

Palabras clave: endófitos, eficiencia en el uso del agua, formaciones xerofíticas, restauración.

\section{INTRODUCCIÓN}

La degradación y la desertificación son uno de los problemas ambientales más serios en la actualidad (MEA 2005), tanto para la conservación de la biodiversidad como para la sustentabilidad de las poblaciones rurales (UNEP 1994). Uno de los ecosistemas más degradados son las formaciones xerofíticas, donde más del $20 \%$ de su superficie se encuentra degradada (Squeo et al. 2007). Las formacio- nes xerofíticas se encuentran constituidas por especies autóctonas, preferentemente arbustivas o suculentas, y según los modelos predictivos de cambio climático, serían uno de los sistemas más sensibles y afectados por los procesos de desertificación (CONAMA 2006, IPCC 2013).

La restauración ecológica es entendida como la actividad deliberada que acelera la recuperación de un sistema degradado por acción directa o indirecta de la actividad humana (Pueyo et al. 2008). La restauración de las forma- 
ciones xerofíticas es estimulada por el establecimiento de plántulas de árboles y arbustos, pero la baja disponibilidad hídrica hace que esta medida sea poco exitosa y extremadamente costosa (Whitford 2002). Se ha demostrado que en estos ambientes, aumentos en la disponibilidad hídrica o una mayor eficiencia en el uso del agua mejorarían el reclutamiento de especies (Holmgren y Scheffer 2001).

La aplicación de hongos que se asocian a raíces ha sido reportada como una exitosa estrategia para mantener o mejorar el desempeño ecofisiológico y supervivencia en diferentes especies de arbustos y árboles (Mei y Flinn 2010). Un ejemplo de estos hongos son los llamados "endófitos", los cuales residen dentro del tejido radicular de su hospedero, comprendiendo un grupo filogenéticamente diverso, con efectos positivos en su hospedero (Jumpponen y Trappen 1998). En ambientes extremos con condiciones climáticamente estresantes (e.g., estrés hídrico), los endófitos han mostrado proporcionar beneficios en términos de supervivencia y crecimiento a la vegetación que se asocia a ellos (Bayman et al. 2002). Un ejemplo de ambientes estresantes es la Antártida, la cual se caracteriza por poseer bajas temperaturas y un marcado déficit hídrico y nutricional (Chwedorzewska 2009).

Por lo anterior, sería esperable que endófitos asociados a raíces de plantas antárticas puedan conferir una ventaja a plantas de otras especies que estén expuestas a condiciones estresantes y que formen asociaciones con ellos. En el presente trabajo, el objetivo es evaluar la supervivencia y la eficiencia en el uso del agua en presencia de endófitos antárticos en tres especies nativas de las formaciones xerofíticas del norte de Chile.

\section{MÉTODOS}

Aislamiento de endófitos. Individuos adultos de Deschampsia antarctica Desv. (Poaceae) fueron colectados en la isla Rey Jorge, islas Shetland del sur (62 $62^{\circ} \mathrm{S}$; $\left.50^{\circ} 28^{\prime} \mathrm{O}\right)$ como fuente de endófitos antárticos. Raíces frescas de 25 individuos fueron cortadas en trozos de $1 \mathrm{~cm}$, esterilizadas con etanol $70 \%$ e hipoclorito de sodio $2 \%$, para posteriormente ser lavadas con agua destilada esterilizada (ver Collao et al. 1996). Cada fragmento de raíz fue depositado en una placa Petri con medio de agar dextrosa (PDA, Difco, USA), y cloranfenicol $100 \mathrm{~mL} \mathrm{~L}^{-1}$. Cada placa fue incubada por 60 días a 4 y $18^{\circ} \mathrm{C}$, y las colonias formadas fueron transferidas nuevamente a PDA y almacenadas a $4{ }^{\circ} \mathrm{C}$ hasta su utilización. No se evidenciaron diferencias en la riqueza de colonias de endófitos entre ambas temperaturas, por la que se utilizaron sólo aquellas incubadas a los $18{ }^{\circ} \mathrm{C}$ debido a que presentaron una mayor abundancia.

Experimento de supervivencia. Para evaluar el efecto de la inoculación con hongos endófitos sobre la supervivencia de especies nativas: Puya berteroniana Mez. (Bromeliaceae), Flourensia thurifera Mol. (Asteraceae) y Senna cumingii Hook. et Am. (Fabaceae), se realizó un experimento de su- pervivencia en el sector de "Quebrada las Vacas" cerca del Parque Nacional "Bosque Fray Jorge" ( $30^{\circ} 41^{\prime}$ 'S; $\left.71^{\circ} 37^{\prime} \mathrm{O}\right)$ a $85 \mathrm{~km}$ al sur de la ciudad de La Serena. Plántulas de cada especie se produjeron desde semillas germinadas en placas Petri con papel absorbente y con un antimicótico de amplio espectro (Benlate, Farmax S.A., Wilmington, DE, USA). Las semillas utilizadas fueron colectadas en diferentes sitios de la región de Coquimbo y mantenidas en el invernadero del Centro de Estudios Avanzados en Zonas Áridas (CEAZA; 29 S). Una vez que cada plántulas tuviese al menos dos hojas verdaderas se transplantaron a maceteros de $1.000 \mathrm{~cm}^{3}$ con una mezcla de arena:suelo del sitio de estudio 1:1. El suelo del sitio de estudio fue previamente autoclavado $\left(120^{\circ} \mathrm{C}\right.$; $\left.2 \mathrm{~h}\right)$ para eliminar la presencia de otros microorganismos que pudieran afectar los resultados. Las plántulas se manutuvieron en invernadero durante 45 días bajo condiciones naturales de luz y temperatura (1.324 mmol m $\mathrm{m}^{-2} \mathrm{~s}^{-1} \pm 243 ; 20^{\circ} \mathrm{C} \pm 4$ ), y con riego diario de $75 \mathrm{~cm}^{3}$ por individuo.

Las plántulas de cada especie fueron divididas en cuatro tratamientos con cuatro réplicas de cinco individuos de cada especie: (i) $100 \%$ de agua (150 $\mathrm{cm}^{3}$ por 3 días); (ii) $70 \%$ de agua (100 $\mathrm{cm}^{3}$ por 3 días); (iii) $100 \%$ de agua + la presencia de endófitos; y (iv) $70 \%$ de agua + la presencia de endófitos. Los $150 \mathrm{~cm}^{3}$ de agua, fue considerada como el $100 \%$, ya que es la cantidad de agua mínima que permitió alcanzar el máximo de crecimiento en dos de las tres especies utilizadas en un ensayo previo realizado en el invernadero del CEAZA (Molina-Montenegro, datos no publicados). La inoculación con endófitos se realizó en dos oportunidades distanciadas por 15 días con una mezcla concentrada de esporas (1000 esporas $\mathrm{g}^{-1}$ ) de los dos endófitos más abundantes (40 y $45 \%$ ) que fueron aislados desde las raíces de $D$. antarctica. Se seleccionaron tres individuos al azar para comprobar que la simbiosis entre la mezcla de endófitos y las raíces fuera efectiva.

Las plántulas de cada especie ( $n$ total $=240$ plántulas) con sus respectivos maceteros fueron asignadas a uno de los cuatro tratamientos previamente descritos y dispuestos con una distancia de $30 \mathrm{~cm}$ entre cada macetero, en un marco de plantación de $3 \times 3 \mathrm{~m}$, donde las plántulas de las tres especies fueron mezcladas. Las réplicas de cada tratamiento estuvieron distanciadas al menos por $10 \mathrm{~m}$ de otra réplica de cualquier tratamiento. El experimento fue monitoreado por 12 meses, y la supervivencia fue registrada semanalmente durante los primeros tres meses y mensualmente en los meses restantes. Considerando que el éxito de reintroducción depende principalmente del número de individuos que efectivamente se logran establecer, mediante un análisis de varianza de una vía (ANDEVA) se comparó la supervivencia de cada especie sometidas a los cuatro tratamientos al final del experimento. Posteriormente, cuando el ANDEVA indicó que hubo efectos significativos de los tratamientos, mediante una prueba $a$ posteriori (Tukey) se identificaron los tratamientos diferentes entre sí. 


\section{RESULTADOS}

En general, la supervivencia en las tres especies fue mayor con la presencia de endófitos y con mayor adición de agua (figuras 1A, 1B y 1C). Para Flourensia thurifera la supervivencia fue significativamente mayor cuando recibió el $100 \%$ de agua y la inoculación de endófitos $\left(\mathrm{F}_{3,12}=16,80\right.$; $P<0,001$ ), seguida por el tratamiento que recibió el $100 \%$ de agua y el de $75 \%$ de agua más endófitos (figura 1A). Finalmente, las plántulas asignadas al tratamiento de $75 \%$ de agua y sin la adición de endófitos presentaron significativamente el menor porcentaje de supervivencia (figura 1A). De manera similar, la supervivencia de Puya berteroniana fue significativamente mayor en los tratamientos con endófitos (100 y $75 \%$ ), seguido por el tratamiento de $100 \%$ de agua, y finalmente por aquel de $75 \%$ y sin la adición de endófitos $\left(\mathrm{F}_{3,12}=19,33 ; P<0,001\right.$; figura 1B). La supervivencia de Senna cumingii fue significativamente mayor en los tratamientos con $100 \%$ de agua con y sin la presencia de endófitos $\left(\mathrm{F}_{3,12}=14,72 ; P<0,001\right)$, seguido por el tratamiento con $75 \%$ de agua más endófitos, y finalmente por el tratamiento de $75 \%$ de agua sin endófitos (figura 1C).

\section{DISCUSIÓN}

Los resultados muestras que la presencia de hongos endófitos aislados de raíces de plantas antárticas aumentó el porcentaje de supervivencia y mejoró la eficiencia en el uso del agua de especies que habitan zonas áridas. De esta manera, podría sugerirse que el uso de hongos provenientes de la Antártica sería una herramienta efectiva para programas de reintroducción en zonas con estrés hídrico como la zona norte de Chile. Esto último es de vital importancia ya que precisamente en estas zonas áridas se encuentran las formaciones xerofíticas de alto valor ecológico caracterizadas por su alto endemismo (ver Squeo et al. 2001).

Chile central ha sido incluido dentro de los 25 "hotspots" de biodiversidad a nivel mundial (Myers et al. 2000). Dentro de esta área de alta biodiversidad, la región de Coquimbo representa el límite septentrional. Investigaciones recientes indican que, en promedio, la biodiversidad aumenta la estabilidad de los ecosistemas (e.g., Chalcraft 2013). Por lo tanto, si la alta biodiversidad favorece la estabilidad ecológica, la acelerada pérdida de especies puede desestabilizar o inclusive llevar al colapso a ecosistemas completos (McCann 2000).

El conseguir un alto reclutamiento natural o el realizar programas de reintroducción podrían aumentar la riqueza y diversidad, y por ende la estabilidad de las formaciones xerofíticas. No obstante, el reclutamiento natural en zonas áridas es bajo debido principalmente a las condiciones abióticas de ese ecosistema (Harrington 1991). Por otro lado, los programas de reintroducción de especies nativas son poco exitosos debido al alto costo y al bajo porcentaje de plantas finalmente establecidas. De esta manera, el

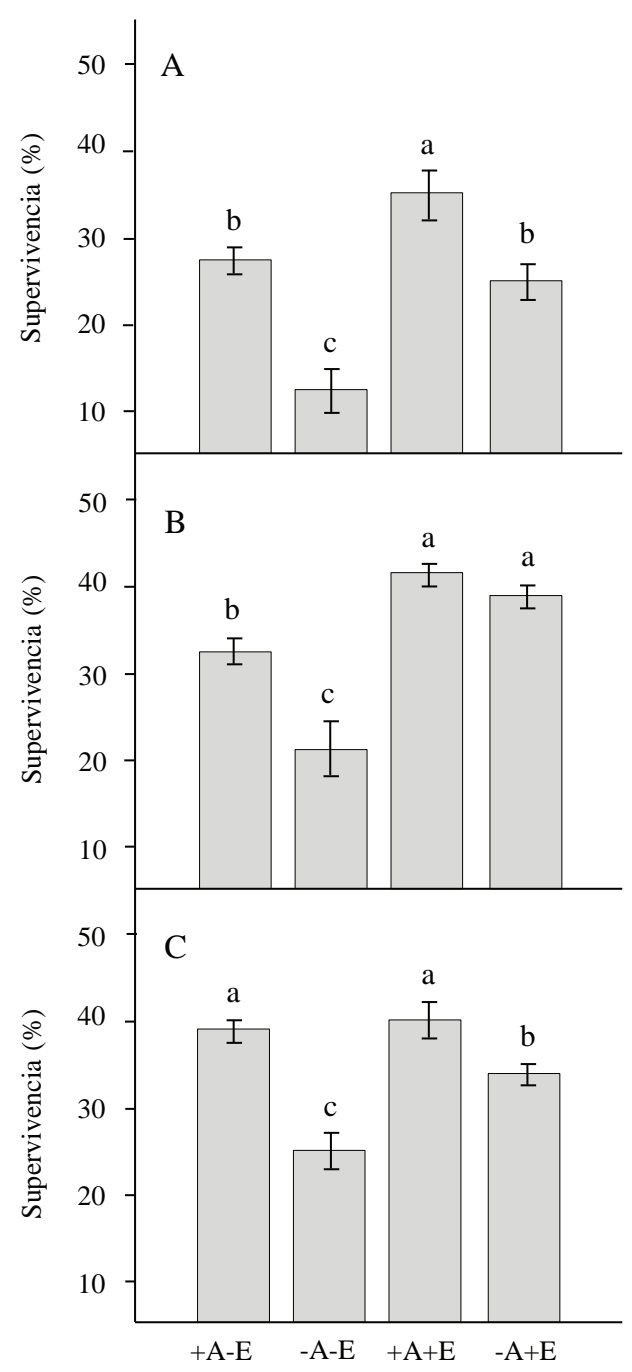

Figura 1. Porcentaje de supervivencia de Flourensia thurifera (A), Puya berteroniana (B) y Senna cumingii (C) a los 12 meses de exposición a tratamientos con diferentes cantidades de adición de agua $(+\mathrm{A}$ y $-\mathrm{A})$ y con presencia y ausencia de hongos endófitos (+E y -E) aislados desde plantas antárticas. Diferentes letras corresponden a diferencias significativas entre tratamientos, prueba a posteriori Tukey; $\alpha=0,05$.

Survival percentage of Flourensia thurifera (A), Puya berteroniana (B) and Senna cumingii (C) after 12 months of exposition to treatments with different amounts of water addition $(+\mathrm{A} y-\mathrm{A})$, and with or without the presence of fungal-endophytes $(+E \mathrm{y}-\mathrm{E})$ isolated from antarctic plants. Different letters correspond to significant differences among treatments, a posteriori Tukey test; a $=0.05$.

presente trabajo podría ser una solución biotecnológica potencial para aumentar el éxito de reintroducción, con menores costos asociados. Por un lado, la aplicación de endófitos aislados de ecosistemas extremos como lo es la Antártica permitió aumentar significativamente el éxito de supervivencia en dos de las tres especies utilizadas. Por otro lado, la inoculación con endófitos antárticos permitió compensar la disminución en la adición de agua, alcanzando una supervivencia similar que el tratamiento con el 
$100 \%$ del agua pero sin endófitos. De esta manera, la aplicación de endófitos permitiría aumentar la supervivencia con el máximo de agua o mantener la misma supervivencia pero con una menor cantidad de agua invertida.

Muchos estudios sugieren que en zonas áridas y semiáridas el agua sería el principal factor limitante para el desempeño de las plantas (Padilla y Pugnaire 2006, Larrea-Alcázar y Soriano 2008). Dentro de los efectos positivos que tiene la mayor disponibilidad hídrica en especies de zonas áridas, destacan el aumento en el porcentaje de supervivencia, aumento en el crecimiento y acumulación de biomasa, mayor producción de semillas y un mejor desempeño fisiológico evidenciado a través de la tasa fotosintética (Gutiérrez et al. 1997, Pugnaire et al. 2004). Por ejemplo, Atala et al. (2012) mostraron por un lado que la inoculación de endófitos a raíces de Pinus radiata $\mathrm{D}$. Don. mejora tanto el crecimiento vegetativo, biomasa, así como la supervivencia de los individuos, y por otro lado, que la presencia de endófitos permite compensar el estrés hídrico. De esta manera, la inoculación con endófitos para maximizar el éxito de establecimiento en especies nativas como en aquellas de importancia económica, podría ser una potencial solución biotecnológica con amplio espectro de aplicación.

Si bien en el presente estudio no se realizó una identificación molecular de los endófitos utilizados, análisis preliminares, tanto morfológicos como enzimáticos, sugieren que podrían ser Ascomycete de los géneros Geomyces y Lecanicillium, los cuales han sido relacionados con tolerancia abiótica y biótica, respectivamente (Hayes 2012, Jackson et al. 2012). Por ejemplo, Geomyces pannorum has sido señalado como un endófitos que mejora la tolerancia al estrés por frío y sequía en plantas antárticas (Rosa et al. 2010). Por otro lado, Jackson et al. (2012) documentaron que la presencia de Lecanicillium lecanii disminuye el ataque de insectos a las raíces. Si bien, estudios más específicos deberían ser conducidos para probar estas hipótesis, no se descarta que los efectos positivos de la inoculación con endófitos antárticos puedan operar de manera simultánea a través de la tolerancia biótica como abiótica.

Los actuales modelos de cambio climático sugieren que la zona norte de Chile estará afectada por prolongados e intensos periodos de sequía, lo cual aumentará la desertificación (CONAMA 2006). Este nuevo escenario climático afectará tanto a las especies presentes como a los programas de reintroducción, volviéndolos más costosos y menos exitosos. Los resultados muestran un significativo y positivo efecto de la presencia de los endófitos tanto en la supervivencia como en la inversión hídrica. Por lo tanto, la aplicación de endófitos antárticos podría comenzar a considerarse para proyectos futuros y programas de reintroducción ecológica en las zonas áridas y semi-áridas de Chile.

\section{AGRADECIMIENTOS}

Agradecemos al proyecto INACH G_22_12 por proveer el material biológico para aislar los endófitos.

\section{REFERENCIAS}

Atala C, E Muñoz-Caponi, G Pereira, E Navarrete, R Oses, MA Molina-Montenegro. 2012. Impact of mycorrhizae and irrigation in the survival of seedlings of Pinus radiata D. Don subject to drought. Gayana Botánica 69: 296-304.

Bayman P, EJ González, JJ Fumero, RL Tremblay. 2002. Are fungi necessary? How fungicides affect growth and survival of the orchid Lepanthes rupestris in the field. Journal of Ecology 90: 1002-1008.

Chalcraft DR. 2013. Changes in ecological stability across realistic biodiversity gradients depend on spatial scale. Global Ecology and Biogeography 22: 19-28.

Chwedorzewska KJ. Terrestrial Antarctic ecosystems in the changing world: An overview. Polish Polar Research 3: 263-276.

Collado J, G Platas, I González, F Peláez. 1999. Geographical and seasonal influences on the distribution of fungal endophytes in Quercus ilex. New Phytologist 144: 525-532.

CONAMA (Comisión Nacional para el Medio Ambiente, CL). 2006. Estudio de la variabilidad climática en Chile para el siglo XXI. Departamento de Geofísica, Facultad de Ciencias Físicas y Matemáticas. Santiago, Chile. Universidad de Chile. 63 p.

Gutiérrez JR, PL Meserve, S Herrera. 1997. Effects of small mammals and vertebrate predators on vegetation in the Chilean semiarid zone. Oecologia 109: 398-406.

Harrington GN. 1991. Effect of soil moisture on shrub seedling survival in a semi-arid grassland. Ecology 72:1138-1149.

Hayes MA. 2012. The Geomyces fungi: ecology and distribution. BioScience 62: 819-823.

Holmgren M, M Scheffer. 2001. El Niño as a window of opportunity for the restoration of degraded arid ecosystems. Ecosystems 4: 151-159.

IPCC (Intergovernmental Panel on Climate Change, CH). 2013. Climate Change 2013: The Physical Science Basis. Disponible en http://www.ipcc.ch/report/ar5/wg1/.

Jackson D, Skillman J, Vandermeer J. 2012. Indirect biological control of the coffee leaf rust, Hemileia vastatrix, by the entomogenous fungus Lecanicillium lecanii in a complex coffee agroecosystem. Biological Control 61: 89-97

Johnson D, F Martin, JWG Cairney, IC Anderson. 2012. The importance of individuals: intraspecific diversity of mycorrhizal plants and fungi in ecosystems. New Phytologist 194: 614-628.

Jumpponen A, Trappe JM. 1998. Dark septate endophytes: a review of facultative biotrophic root-colonizing fungi. New Phytologist 140:295-310.

Larrea-Alcázar DM, PJ Soriano. 2008. Columnar cacti-shrub relationships in an Andean semiarid valley in western Venezuela. Plant Ecology 196: 153-161.

McCann KS. 2000. The diversity - stability debate. Nature 405: 220-227.

Mei C, Flinn BS. 2010. The use of beneficial microbial endophytes for plant biomass and stress tolerance improvement. Recent Patents on Biotechnology 4:81-95

Millennium Ecosystem Assessment. 2005. Ecosystems and Human Well-being. Synthesis. Washington, DC, USA. Island Press. 137 p.

Myers N, RA Mittermeier, CG Mittermeier, DA Da Fonseca, J Kent. 2000. Biodiversity hotspots for conservation priori- 
ties. Nature 403: 853-858.

Padilla FM, FI Pugnaire. 2006. The role of nurse plants in the restoration of degraded environments. Frontiers in Ecology and Environment 4: 196-202.

Pueyo Y, CL Alados, B García-Ávila, S Kéfi, M Maestro, M Rietkerk. 2008. Comparing direct abiotic amelioration and facilitation as tools for restoration of semiarid grasslands. Restoration Ecology 17: 908-916.

Pugnaire FI, C Armas, F Valladares. 2004. Soil as a mediator in plant-plant interactions in a semi-arid community. Journal of Vegetation Science 15: 85-92.

Rosa LH, ML Almeida-Vieira, I Furtado, CA Rosa. 2010. Endophytic fungal Community associated with the dicotyledonous plant Colobanthus quitensis (Kunth) Bartl. (Caryophyllaceae) in Antarctica. FEMS Microbiology Ecology 73:
178-189.

Squeo FA, Arancio G, Gutiérrez JR. 2001. Libro Rojo de la Flora Nativa de la Región de Coquimbo y de los Sitios Prioritarios para su Conservación. La Serena, Chile. Ediciones de la Universidad de La Serena. 361 p.

Squeo FA, M Holmgren, M Jiménez, L Albán, J Reyes, JR Gutiérrez. 2007. Tree establishment along an ENSO experimental gradient in the Atacama desert. Journal of Vegetation Science 18: 195-202.

UNEP (United Nations Environment Programme). 1994. United Nations Convention to Combat Desertification in those countries experiencing serious drought and/or desertification, particularly in Africa.

Whitford WG. 2002. Ecology of Desert Systems. San Diego, USA. Academic Press. 327 p.

Recibido: 22.02.14

Aceptado: 13.05 .14 
